\title{
Maternal Protein Restriction Alters the Renal Ptger1 DNA Methylation State in SHRSP Offspring
}

\author{
Moe Miyoshi ${ }^{1}$, Masayuki Sato ${ }^{1}$, Kenji Saito ${ }^{1}$, Lila Otani ${ }^{1}$, Katsuhiko Shirahige ${ }^{2}$, \\ Fumihito Miura ${ }^{3} \mathbb{D}$, Takashi Ito ${ }^{3}$, Huijuan Jia ${ }^{1}$ and Hisanori Kato ${ }^{1, *}$ \\ 1 Health Nutrition, Graduate School of Agricultural and Life Sciences, The University of Tokyo, \\ Tokyo 1138657, Japan; monet344myo@gmail.com (M.M.); sato-mas@lion.co.jp (M.S.); \\ kkkj774@yahoo.co.jp (K.S.); nf_lila@yahoo.co.jp (L.O.); ginajhj@gmail.com (H.J.) \\ 2 Research Center for Epigenetic Disease, Institute of Molecular and Cellular Biosciences, \\ The University of Tokyo, Tokyo 1130032, Japan; kshirahi@gmail.com \\ 3 Department of Biochemistry, Kyushu University Graduate School of Medical Sciences, Kyushu University, \\ Fukuoka 8128582, Japan; fumihito@med.kyushu-u.ac.jp (F.M.); tito@med.kyushu-u.ac.jp (T.I.) \\ * Correspondence: akatoq@mail.ecc.u-tokyo.ac.jp; Tel./Fax: +81-3-5841-1607
}

Received: 6 September 2018; Accepted: 28 September 2018; Published: 5 October 2018

\begin{abstract}
We previously reported that maternal protein restriction (LP) during pregnancy increases salt sensitivity in offspring using the Stroke-Prone Spontaneously Hypertensive Rat (SHRSP). In the present study, we focus on DNA methylation profiles of prostaglandin E receptor 1 gene (ptger1), which is known to be associated with hypertension. We evaluated the ptger 1 DNA methylation status via bisulfite sequencing, and analyzed the expression of ptger1-related genes. The results of these analyses showed that, compared to controls, the LP-S offspring exhibited both marked ptger 1 hypermethylation, and significantly increased ptger 1 expression. Moreover, they also exhibited significantly decreased expression of the downstream gene epithelial $\mathrm{Na}^{+}$channel alpha (enack). Interestingly, LP offspring that were provided with a standard water drinking supply (W) also exhibited increased ptger 1 methylation and expression. Together, these results suggest that maternal protein restriction during pregnancy modulates the renal ptger1 DNA methylation state in SHRSP offspring, and thereby likely mediates ptger1 and enac $\alpha$ gene expression to induce salt sensitivity.
\end{abstract}

Keywords: maternal protein restriction; salt sensitivity; DNA methylation

\section{Introduction}

In recent years, the concept that the fetal nutritional state affects the lifetime risk of pathophysiological processes associated with chronic (including especially non-communicable) diseases, (i.e., the 'Fetal Origins of Adult Disease (FOAD)', and 'Developmental Origins of Health and Disease $(\mathrm{DOHaD})$ ' concepts originally proposed by Barker in the 1990s [1-3]), has become widely accepted. For example, several studies have demonstrated that low birth weight caused by maternal undernutrition is strongly associated with childhood obesity, diabetes, and cardiovascular disorders [4-7].

Epigenetic modifications are chemical changes to DNA and/or histones that regulate gene expression without altering the DNA sequence. Previous studies have shown that DNA methylation changes tend to be retained in chromatin across generations [8]; thus, they have been proposed as possible key mechanisms that underlie $\mathrm{DOHaD}$ [9-11].

The Stroke-Prone Spontaneously Hypertensive rat (SHRSP) naturally develops stroke as a result of harboring a heredity factor that causes hypertension, and has accordingly been widely used as a research model for cardiovascular diseases. In our previous study using the SHRSP, protein restriction 
during pregnancy significantly increased the rate of salt-sensitive hypertension and early death among offspring that were provided with a $1 \%$ saline drinking solution, but not among those that were administered tap water [12,13]. Similarly, the production of aldosterone (a key factor in the renin-angiotensin-aldosterone system (RAAS)) was increased, and type II angiotensin receptor protein levels in the kidney and adrenal gland were markedly affected in the offspring of dams that underwent protein restriction during pregnancy $[13,14]$. Therefore, in the present study, we hypothesized that epigenetic changes may modulate salt sensitivity after maternal protein restriction, and accordingly attempted to identify novel target genes via a DNA methylation analysis.

\section{Materials and Methods}

\subsection{Ethics Statement and Animal Conditions}

The present study was approved, and conducted in strict accordance with the guidelines stipulated by the Animal Usage Committee of the Graduate School of Agricultural and Life Sciences, at The University of Tokyo, (Approval No. P09-376). Rats were maintained at $22 \pm 1{ }^{\circ} \mathrm{C}$, in $60 \% \pm 5 \%$ humidity, and in a $12 \mathrm{~h}$ light (8:00 to 20:00)/dark cycle.

\subsection{Animal Experiment 1: Water-Drinking Offspring}

Eight-week-old male and female SHRSP/Izm rats were obtained from the Disease Model Cooperative Research Association (Kyoto, Japan). After acclimatizing to the stipulated environmental conditions for 1 week, virgin female SHRSP rats (body weight, 169-183 g; blood pressure, 173-186 mmHg) were mated to a single stud male (body weight, 241-260 g; blood pressure, 175-187 mmHg). Pregnancy was confirmed by the presence of a semen plug. Pregnant dams were randomly allocated to be fed a $20 \%(\mathrm{CN})$ or $9 \%$ casein diet (LP) during pregnancy (Table 1). After delivery, all dams were fed a commercial diet (MF diet; Oriental Yeast, Tokyo, Japan). At the fourth postnatal week, male pups were separated from the dams, and provided with ad libitum access to drinking water $(\mathrm{W})$ and a commercial diet. Finally, 12-week-old male (CN-W or LP-W) offspring were culled, and their kidney, brain, and heart samples were collected.

Table 1. Diet composition.

\begin{tabular}{ccc}
\hline Component (\%) & CN (20\% Casein) & LP (9\% Casein) \\
\hline Casein & 20.0 & 9.0 \\
Corn starch & 66.8 & 77.8 \\
DL-Methionine & 0.2 & 0.2 \\
Soy bean oil & 5.0 & 5.0 \\
Vitamin mixture * & 1.0 & 1.0 \\
Mineral mixture * & 4.0 & 4.0 \\
Cellulose powder & 3.0 & 3.0 \\
\hline rescription (Oriental Yeast Co., Ltd., Tokyo, Japan). CN, 20\%-Casein diet; LP, 9\%-Casein diet.
\end{tabular}

\subsection{Animal Experiment 2: Salt-Loading Offspring}

Virgin female SHRSP rats (body weight, 152-165 g; blood pressure, 161-176 mmHg) were mated to a stud male (body weight, 217-227 g; blood pressure, 153-173 mmHg). The rats were maintained as described in Animal Experiment 1 during pregnancy and lactation period. Male offspring were separated from the dams at the fourth postnatal week provided with ad libitum access to drinking water and commercial diet, and then they were administered a $1 \%$ saline solution (S) during weeks 10-12 after birth (CN-S or LP-S). 


\subsection{Blood Pressure Measurement}

Blood pressure was measured as described in our previous report [12]. Briefly, blood pressure measurements were taken from the tail (BP-98A; Softron, Tokyo, Japan) of offspring at 10, 11, and 12 weeks after birth, without sedation.

\subsection{Biochemical Analysis}

Urine samples were collected over a 4-day period from 11-week-old offspring, and the urinary albumin excretion level was measured using a Rat Albumin ELISA kit (AKRAL-120; Shibayagi, Gunma, Japan). Plasma was collected from 12-week-old offspring, and the aldosterone concentration was measured using a Detect $X^{\circledR}$ Aldosterone Enzyme Immunoassay kit (Arbor Assays, Michigan, MI, USA).

\subsection{Quantitative Real-Time PCR}

The total RNA from kidney samples was extracted using a NucleoSpin ${ }^{\circledR}$ TriPrep kit (Takara Bio, Tokyo, Japan), and reverse transcribed to cDNA using PrimeScript ${ }^{\mathrm{TM}}$ RT Master Mix (Perfect Real Time) (Takara Bio, Tokyo, Japan). Gene segments were amplified from the synthesized cDNA using the Thermal Cycler Dice Real Time System TP800 (Takara Bio, Tokyo, Japan), SYBR ${ }^{\circledR}$ Premix Ex Taq ${ }^{\mathrm{TM}}$ (Tli RNaseH Plus) (Takara Bio, Tokyo, Japan), and appropriate primers (Supplementary Information (SI) Table S1). mRNA levels were normalized to those of $18 S$ rRNA, and expressed as fold-change values.

\subsection{Analysis of DNA Methylation Profile}

To determine the target gene for DNA methylation analysis, we conducted an exploratory genome-wide methylation (methylome) analysis $(n=1)$ and found that one of the genes located within/adjacent to the differentially methylated regions identified in the salt-sensitized offspring was the prostaglandin E receptor 1 gene (ptger1).

Then we focused on the methylation status of CpG sites (CpGs) within the prostaglandin E receptor 1 (ptger1) gene (region from transcription initiation site; +1315 to $+1614,300 \mathrm{bp}$ ) and examined it via bisulfite sequencing. Briefly, renal genomic DNA was bisulfite-converted using a BisulFlash $^{\mathrm{TM}}$ DNA Modification Kit (Epigentek, Farmingdale, NY, USA), and amplified using EpiTaq $^{\mathrm{TM}}$ HS (for bisulfite-treated DNA) (Takara Bio, Tokyo, Japan) and ptger1 primers (sense, 5'-AATATATTTGTGGTGTTGTTAATAGG-3'; antisense, 5' -ACCAAAAAAAACCATACAACC-3'). The amplified PCR products were separated via 1\% Agarose-ME (Nacalai Tesque, Inc., Tokyo, Japan) gel electrophoresis, and purified using the Wizard SV Gel and PCR Clean-Up system (Promega, Madison, WI, USA). They were then ligated into the pGEM-T easy vector (Promega, Madison, WI, USA), and transformed into Escheria coli DH5 $\alpha$ competent cells (Sigma-Aldrich, Saint Louis, MO, USA). Plasmid DNA was isolated using a Gene Elute ${ }^{\mathrm{TM}}$ Plasmid MiniPrep kit (Sigma-Aldrich, Saint Louis, MO, USA). Clones were commercially sequenced by Eurofins Genomics (Tokyo, Japan), and sequencing data were analyzed using Quantification tool for Methylation Analysis software (http: / / quma.cdb.riken.jp /) (Last updated at Jul 19 00:30:00 JST 2014) (RIKEN Center for Developmental Biology, Kobe, Japan).

\subsection{Statistical Analysis}

Values were expressed as the mean \pm standard error. The statistical significance of data produced by Animal Experiments 1 and 2 was assessed using a one-way analysis of variance (ANOVA), followed by a Student's t-test. A $p$ value $<0.05$ was considered to indicate statistical significance. 


\section{Results}

\subsection{Blood Pressure of Offspring from 10 to 12 Weeks after Birth}

No significant differences were observed between the liquid consumption of the CN-W and CN-S compared to the LP-W and LP-S offspring, respectively, between 10 and 12 weeks after birth (SI Tables S2 and S3). Similarly, the blood pressures of the CN-W and LP-W offspring were not significantly different between weeks 10 to 12 after birth (Figure 1a). In contrast, the blood pressure of the LP-S offspring was significantly elevated compared to that of the CN-S offspring at 12 weeks-of-age (Figure 1b).

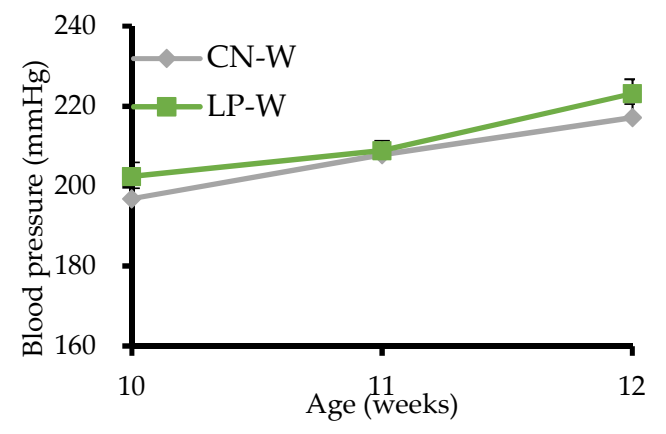

(a)

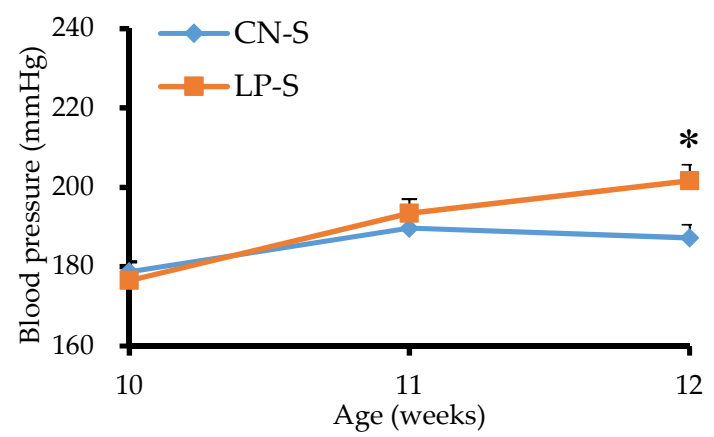

(b)

Figure 1. Effect of low protein intake during pregnancy on the blood pressure of offspring. Blood pressure changes from 10 to 12 weeks after birth in (a) CN-W and LP-W (Animal Experiment 1), and (b) $\mathrm{CN}-\mathrm{S}$ and LP-S (Animal experiment 2) offspring. Values are expressed as the mean \pm standard error $(n=7-8) .{ }^{*} p<0.05$ vs. the CN-S group, according to a Student's $t$-test. Offspring were exposed to either an intrauterine environment of $20 \%(\mathrm{CN})$-feeding mothers or that of $9 \%$ (LP)-Casein diet-feeding mothers, and provided with either water $(\mathrm{W})$ or $1 \%$ saline drinking supply (S).

3.2. Offspring Organ Weight, and Urinary Albumin Excretion, and Plasma Aldosterone Levels at 12 Weeks after Birth

The induced changes to maternal protein intake did not affect the final body weight of the offspring (SI Tables S2 and S3). Likewise, all offspring administered drinking water exhibited similar kidney, brain, and heart weights (SI Table S2). In contrast, while the kidney and brain weights exhibited by the two groups of offspring provided with the saline drinking solution were equivalent, the heart weights exhibited by the LP-S offspring were significantly higher than those of the CN-S offspring, (SI Table S3).

No significant difference in urinary volume was observed between the CN-W and LP-W, nor the CN-S and LP-S offspring (SI Tables S2 and S3). However, the urinary albumin excretion level, which is a biomarker for acute kidney injury, was similar in the CN-W and LP-W offspring (Figure 2a), but tended to be higher in the LP-S than the CN-S offspring (Figure 2b). Conversely, while the plasma levels of the pivotal blood pressure regulator aldosterone were similar in the CN-S and LP-S offspring (Figure 2d), they were significantly increased in the LP-W compared to the CN-W offspring (Figure 2c). 


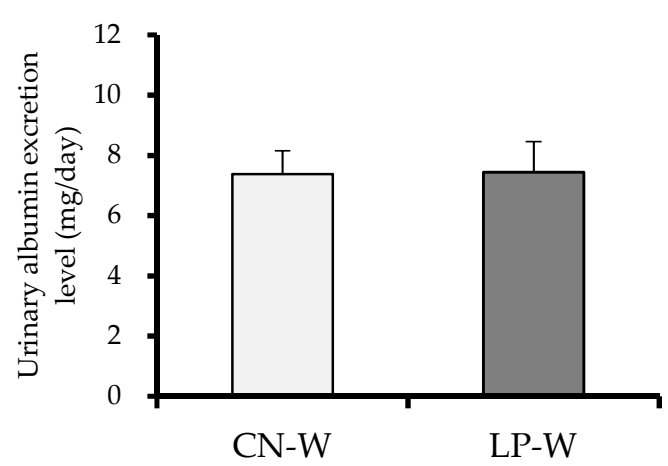

(a)

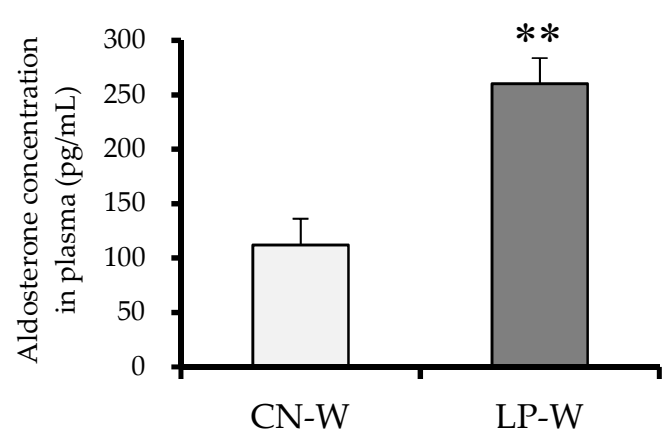

(c)

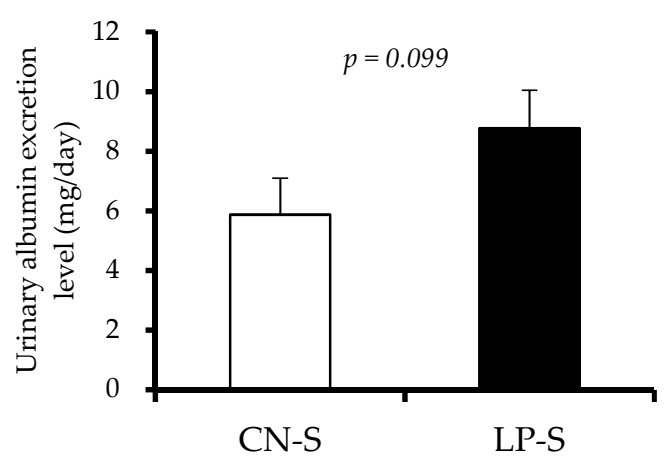

(b)

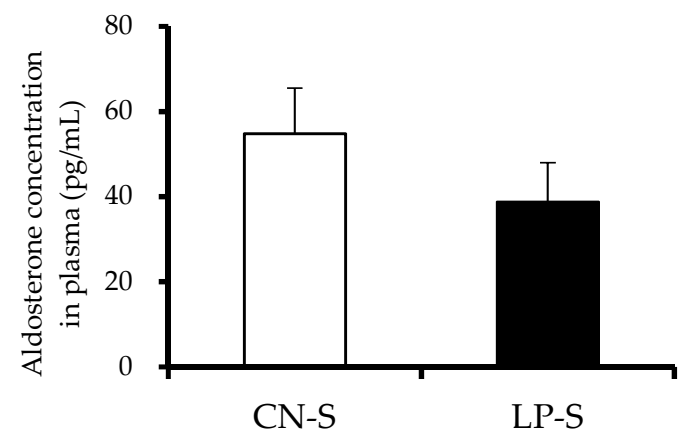

(d)

Figure 2. Effect of low protein intake during pregnancy on the exhibition of renal damage markers by offspring at 12 weeks after birth. (a) Offspring urinary albumin excretion levels (Animal Experiment 1). (b) Offspring urinary albumin excretion levels (Animal Experiment 2). (c) Aldosterone concentration levels in offspring plasma (Animal Experiment 1). (d) Aldosterone concentration levels in offspring plasma (Animal Experiment 2). Values are expressed as the mean \pm standard error $(n=7-8)$. ** $p<0.01$ vs. the CN-W group according to a Student's $t$-test. Offspring were exposed to either an intrauterine environment of $20 \%(\mathrm{CN})$-feeding mothers or that of $9 \%$ (LP)-Casein diet-feeding mothers, and provided with either water $(\mathrm{W})$ or $1 \%$ saline drinking supply (S).

\subsection{DNA Methylation Status in the Ptger1 Gene Region in Offspring Kidney Samples}

First of all, we determined to focus on ptger 1 gene by an exploratory methylome analysis, since ptger1 encodes the EP1 subtype of the prostaglandin E2 (PGE2) receptor, which is known to both mediate algesia, and regulate blood pressure.

Next all four groups of offspring (CN-W, $n=3$; LP-W, $n=3 ; \mathrm{CN}-\mathrm{S}, n=5 ; \mathrm{LP}-\mathrm{S}, n=6)$ were subjected to bisulfite sequencing to investigate the DNA methylation status of the transcribed ptger 1 region (region from transcription initiation site; +1315 to +1614 ), which contains $23 \mathrm{CpGs}$. The overall DNA methylation levels in this region were shown to be higher in the LP-S, and to a lesser extent in the LP-W, than in the CN-S and CN-W offspring, respectively (Figure 3a,b, SI Figure S1). Three CpGs (CpG-13, CpG-20, and CpG-23) were significantly hypermethylated, and two others (CpG-1, and CpG-7) tended to be hypermethylated in the LP-W compared to the CN-W offspring (Fig. 3c). Similarly, five CpGs (CpG-1, CpG-3, CpG-4, CpG-9, and CpG-12) were significantly hypermethylated, and another five (CpG-5, CpG-8, CpG-10, CpG-11, CpG-18) tended to be hypermethylated in the LP-S compared to the CN-S offspring (Figure 3d). 


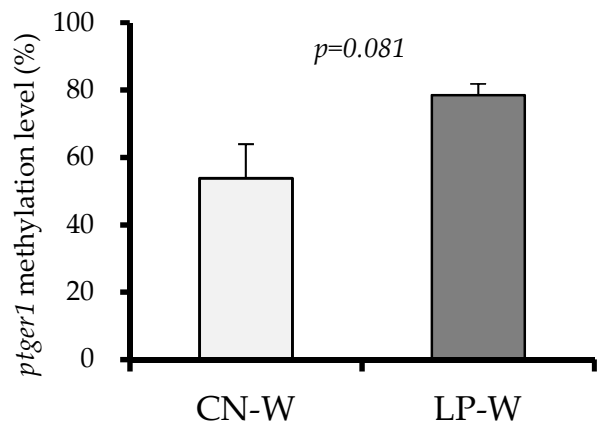

(a)

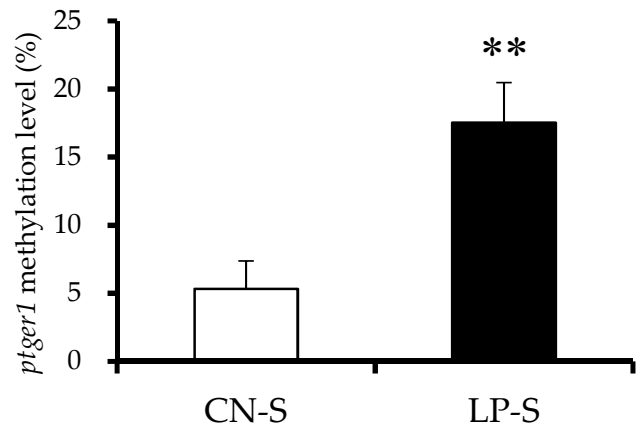

(b)

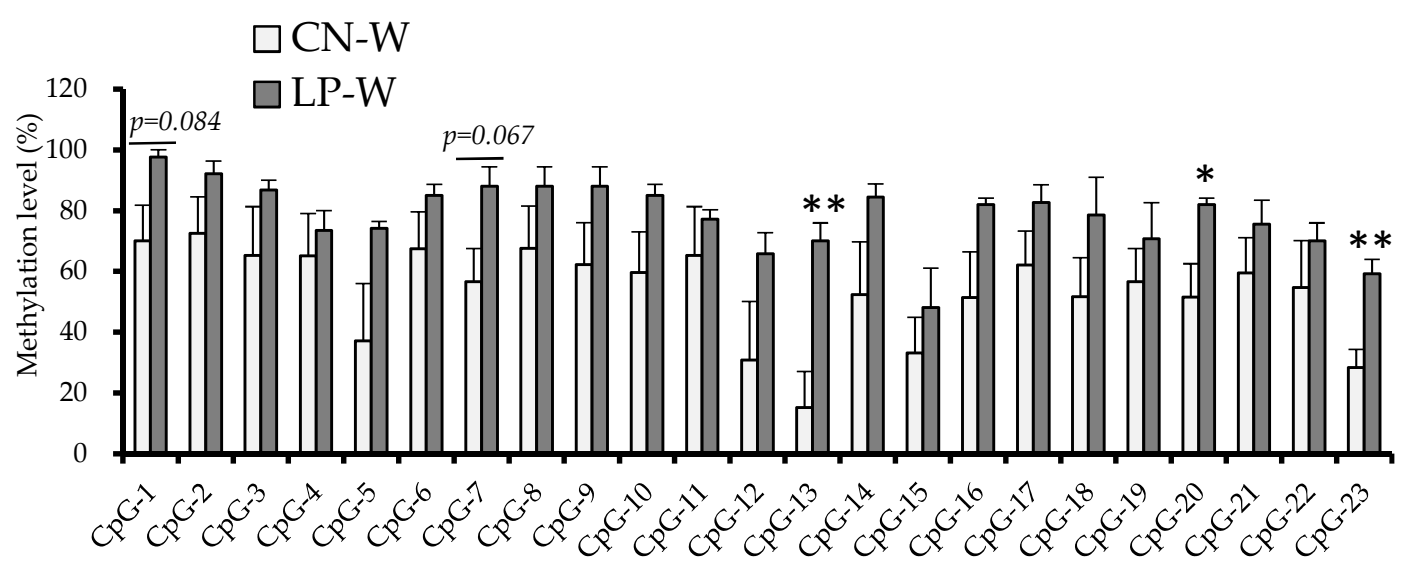

(c)

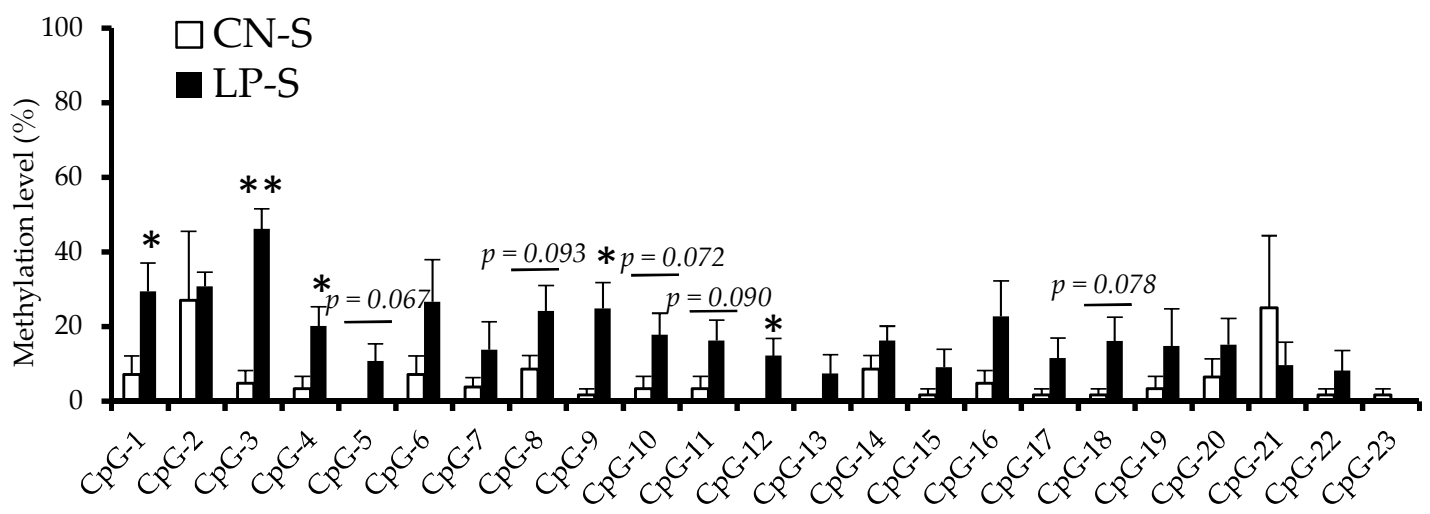

(d)

Figure 3. DNA methylation levels in the transcribed region of the ptger1 gene in offspring kidney samples. (a) The ptger1 total methylation level (Animal Experiment 1). (b) The ptger1 total methylation level (Animal Experiment 2). (c) Methylation levels at 23 ptger1 CpG sites (Animal Experiment 1). (d) Methylation levels at 23 ptger1 CpG sites (Animal experiment 2). Values are expressed as the mean \pm standard error. ${ }^{* *} p<0.01,{ }^{*} p<0.05$ vs. the $\mathrm{CN}-\mathrm{W}$ or CN-S offspring according to a Student's $t$-test. Offspring were exposed to either an intrauterine environment of $20 \%(\mathrm{CN})$-feeding mothers or that of $9 \%(\mathrm{LP})$-Casein diet-feeding mothers, and provided with either water $(\mathrm{W})$ or $1 \%$ saline drinking supply (S).CN-W, $n=3$; LP-W, $n=3 ; \mathrm{CN}-\mathrm{S}, n=5 ; \mathrm{LP}-\mathrm{S}, n=6$. 


\subsection{Offspring Renal Expression Of Ptger1-Related Genes}

Renal ptger 1 expression levels were significantly higher in the LP-S and LP-W than in the CN-S and $\mathrm{CN}-\mathrm{W}$ offspring, respectively (Figure $4 \mathrm{a}, \mathrm{b}$ ). Conversely, expression of the downstream epithelial $\mathrm{Na}^{+}$channel alpha (enac $\alpha$ ) gene was significantly decreased in LP-S compared to CN-S offspring (Figure $4 \mathrm{~d}$ ), but was unchanged between the $\mathrm{CN}-\mathrm{W}$ and LP-W offspring (Figure 4c). The expression of additional genes down- and upstream of ptger1, comprising those that encode the beta (enac $\beta$ ) and gamma (enac $\gamma$ ) epithelial $\mathrm{Na}^{+}$channel subunits, and that which encodes prostaglandin $\mathrm{E}$ synthase 3 (ptges3), respectively, were also examined, and shown to be similar between the various offspring groups (SI Figure S2).

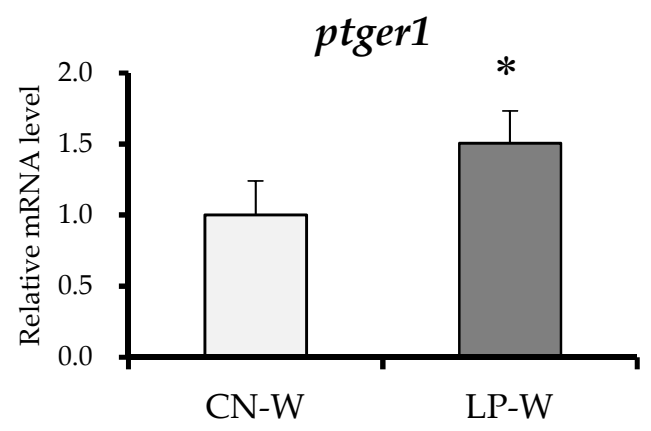

(a)

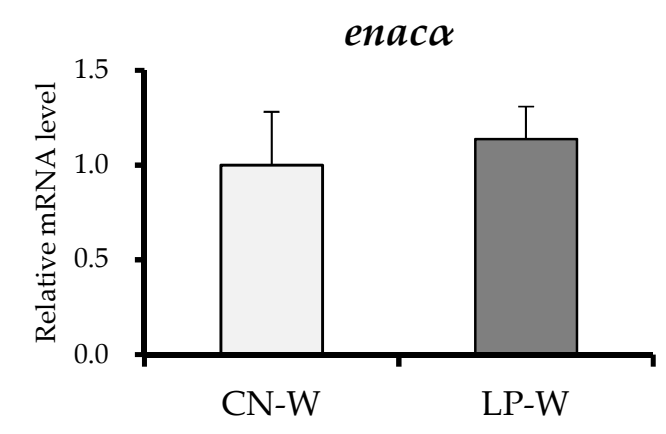

(c)

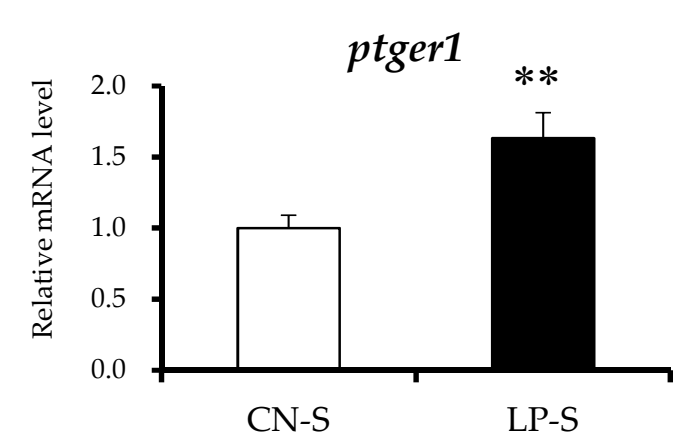

(b)

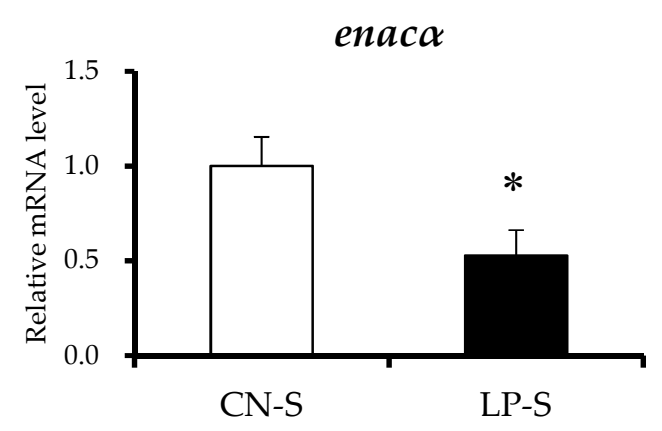

(d)

Figure 4. Relative renal expression of ptger 1 and enac $\alpha$ mRNA. (a) ptger 1 mRNA expression levels (Animal experiment 1). (b) ptger 1 mRNA expression levels (Animal Experiment 2). (c) enac $\alpha$ mRNA expression levels (Animal Experiment 1). (d) enac $\alpha$ mRNA expression levels (Animal Experiment 2). Values are expressed as the mean \pm standard error $(n=7-8) .{ }^{* *} p<0.01,{ }^{*} p<0.05$ vs. the CN-W or CN-S offspring according to a Student's $t$-test. Offspring were exposed to either an intrauterine environment of $20 \%$ (CN)-feeding mothers or that of $9 \%$ (LP)-Casein diet-feeding mothers, and provided with either water $(\mathrm{W})$ or $1 \%$ saline drinking supply (S).

\section{Discussion}

This study is the first to examine the effect(s) of maternal protein restriction on DNA methylation and gene expression in SHRSP offspring. The elevation of the blood pressure and urinary albumin excretion in the 12-week-old LP-S offspring suggests that the salt sensitivity of the offspring was increased in response to the imposed low maternal protein consumption. Consistent with our previous reports, this effect did not impact the blood pressure, nor the urinary albumin excretion of the LP-W (compared to the $\mathrm{CN}-\mathrm{W}$ ) offspring [12,13]. 
From the results of the conducted methylome analysis, we selected ptger 1 for further analysis, due to its known association with hypertension [15]. As discussed, ptger1 encodes the EP1 subtype of the PGE2 receptor, which is one of four PGE2 receptor subtypes (EP1-4) [16]. PGE2 is a critical product of arachidonic acid metabolism in the kidney, and functions to transport both $\mathrm{Na}^{+}$and water, strain vascular smooth muscle, filter glomeruli, and promote renin secretion [17]. In fact, intrarenal PGE2 production has been shown to increase as RAAS activity levels rise [17]. Accordingly, a previous study demonstrated that (orally) administering 10-week-old SHRSP with a selective EP1 antagonist for five weeks was sufficient to cause both decreases in arteriole wounding, transforming growth factor-beta (TGF- $\beta$ ) expression, renal tubule stroma fibrosis, and urinary protein excretion, and an increase in renal creatinine clearance [15]. Thus, PGE2/EP1 signaling is thought to promote renal damage when blood pressure is elevated. Notably, our bisulfate analysis revealed that the ptger 1 methylation status was increased in the LP-S and LP-W compared to the CN-S and CN-W offspring, respectively (Figure 3). This suggests that the renal ptger 1 sequence was hypermethylated in offspring in response to the imposed maternal low-protein intake. In addition, the DNA methylation profile at the analyzed CpGs varied markedly between offspring provided with a saline compared to a normal drinking supply; thus, DNA methylation was affected by the imposed changes to both the maternal dietary protein, and offspring adult salt water intake.

While a number of studies have reported that gene transcription is inactivated by promoter methylation, others have shown it to cause gene expression to increase $[18,19]$. To date, the impact of methylation in transcribed regions on gene expression regulation is not well understood; however, some cases of upregulated gene expression resulting from hypermethylation within transcribed regions have been reported [20,21]. In this study, the ptger 1 DNA methylation profile in LP-W and LP-S offspring was increased by exposure to maternal protein restriction. The same offspring also exhibited significantly increased ptger 1 expression, suggesting that the incurred methylation changes may have upregulated ptger 1 mRNA expression. Thus, ptger1 may be a novel epigenetic marker for salt sensitivity in the presence (or absence) of fetal protein restriction; however, further study is needed to confirm the effects of ptger 1 methylation and the effects of other important factors for expression of ptger1.

According to Guyton et al. [22], hypertension can occur when shifts in the relationship between renal pressure and natriuresis cause an increased salt intake, leading to high blood pressure. Thus, aberrant renal $\mathrm{Na}^{+}$excretion is required for the development of salt sensitivity [22]. The epithelial $\mathrm{Na}^{+}$channel (ENaC) critically regulates renal $\mathrm{Na}^{+}$excretion, and thereby also salt sensitivity [23]. As discussed, there are three homologous ENaC subtypes, comprising the $\alpha$ (the major subtype), $\beta$, and $\gamma$ subtypes [24], and ENaC activity is positively regulated by aldosterone [25]. In fact, enac $\alpha$ expression has been shown to increase in the presence of aldosterone, leading to enhanced $\mathrm{Na}^{+}$ resorption [26]; however, this is prevented by RAAS-induced PGE2/EP1 activation [27]. In the present study, the plasma aldosterone concentration was significantly increased in the LP-W offspring; however, this difference was not accompanied by a change in enaca expression levels. The increased ptger1 mRNA expression in these offspring may have suppressed the effect of aldosterone on enack mRNA induction, thereby preventing the expected aberrant renal $\mathrm{Na}^{+}$excretion and blood pressure elevation. Conversely, the LP-S offspring exhibited reduced enac $\alpha$ expression, likely as a result of their observed increased ptger 1 expression, and unchanged plasma aldosterone concentration. Together, these observations suggest that reduced enac $\alpha$ mRNA levels suppress renal $\mathrm{Na}^{+}$reabsorption, and that aberrant $\mathrm{Na}^{+}$excretion likely mediated the salt sensitivity exhibited by the LP-S offspring.

As the future work of this study, we think that it will be important to reveal whether these ptger 1 and enac $\alpha$ changes are found in other rodent models such as normotensive model rat (e.g., Wistar rat and WKY rat) and mice models because it is unclear if our results are hypertensive model rat specific. In addition, we used kidney as the target organ for elucidation of a mechanism of hypertension, and it will be interesting to analyze other organs like the brain and heart, which we have already collected in 
this study. In consideration of investigating live humans (clinical tests), studies using accessible tissues like blood may be useful.

\section{Conclusions}

The results of the present study suggest that the imposed maternal protein restriction modulated renal ptger1 methylation in the SHRSP offspring, and that the induced ptger1 methylation changes likely altered renal ptger 1 and enaca gene expression to induce salt sensitivity (Figure 5). Further research is needed to confirm these conclusions, and to investigate whether the same mechanisms are important during human development.

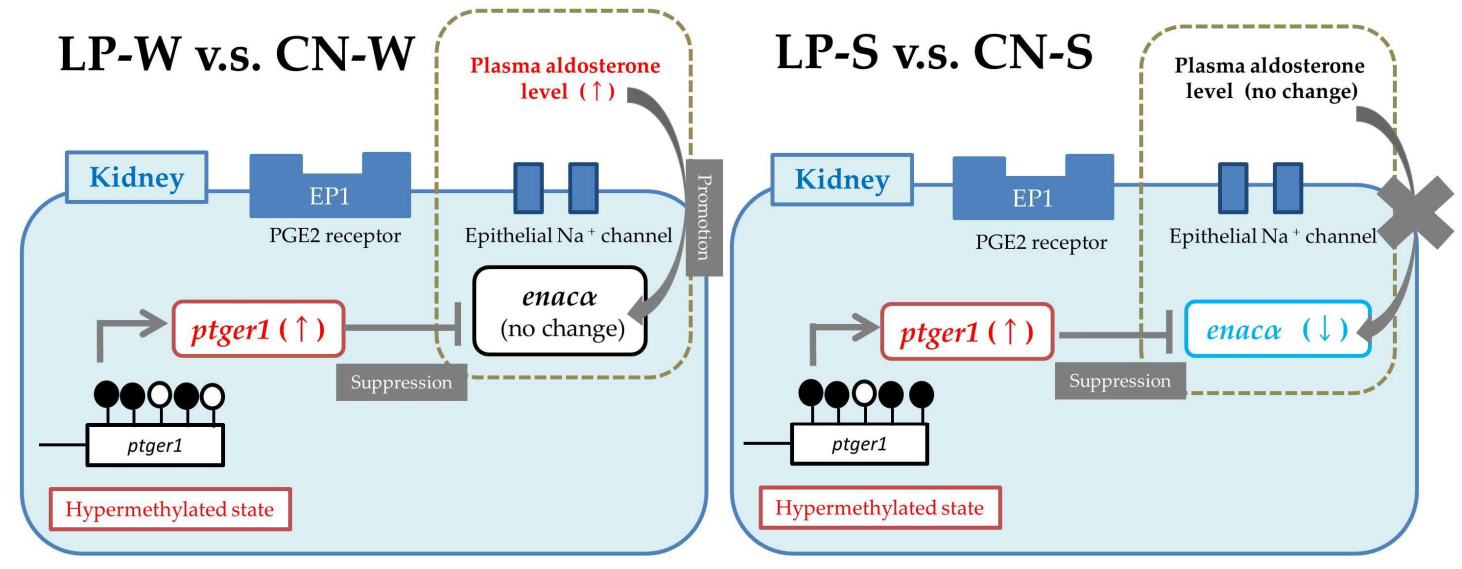

Figure 5. Schematic diagram showing the hypothesized changes to renal $\mathrm{Na}^{+}$transport/homeostasis that were incurred in the Spontaneously Hypertensive Stroke Prone Rat (SHRSP) offspring by the imposed maternal protein restriction.

Supplementary Materials: The following are available online at http://www.mdpi.com/2072-6643/10/10/ 1436/s1, Table S1: Primers used in the real-time (RT)-PCR analysis of ptger1-related gene expression, Table S2: Liquid consumption, urine volume, and organ weights exhibited by offspring provided with drinking water $(\mathrm{W})$, and either a high (CN) or low (LP)-protein diet, Table S3: Liquid consumption, urine volume, and organ weights in exhibited by offspring provided with a $1 \%$ saline drinking solution (S), and either a high $(\mathrm{CN})$ or low (LP)-protein diet, Figure S1: ptger1 DNA methylation status according to the conducted bisulfite-sequencing analysis. CN, 20\%-Casein diet; LP, 9\%-Casein diet; S, 1\% saline drinking solution; W, drinking water, Figure S2: Relative renal mRNA expression of ptger1-related genes. Values are expressed as the mean \pm standard error $(n=7-8)$. CN, 20\%-Casein diet; LP, 9\%-Casein diet; S, 1\% saline drinking solution; $\mathrm{W}$, drinking water.

Author Contributions: M.S. and L.O. conducted the animal breeding, blood pressure measurements, and biochemical (plasma and urine) analyses. M.S., K.S., F.M., and T.I. performed the methylome analysis, and M.M. conducted the DNA methylation state assessments, bisulfite-sequencing, and mRNA expression analyses. M.M. wrote, and H.K. and H.J. revised the paper. All authors reviewed the manuscript.

Funding: This work was supported by JSPS KAKENHI Grant Number JP 24380066.

Acknowledgments: The authors would like to acknowledge the contribution of the various other members of the Health Nutrition laboratory. This work was supported by members of the Research Center for Epigenetic Disease, and the Kyushu University Graduate School of Medical Sciences.

Conflicts of Interest: The corresponding author belongs to a university-community relations laboratory that is associated with Ajinomoto Co., Inc.

\section{References}

1. Osmond, C.; Barker, D.J.; Winter, P.D.; Fall, C.H.; Simmonds, S.J. Early growth and death from cardiovascular disease in women. BMJ 1993, 307, 1519-1524. [CrossRef] [PubMed]

2. Barker, D.J.; Martyn, C.N.; Osmond, C.; Hales, C.N.; Fall, C.H. Growth in utero and serum cholesterol concentrations in adult life. BMJ 1993, 307, 1524-1527. [CrossRef] [PubMed]

3. Barker, D.J. Fetal origins of coronary heart disease. BMJ 1995, 311, 171-174. [CrossRef] [PubMed] 
4. Roseboom, T.J.; van der Meulen, J.H.; Ravelli, A.C.; Osmond, C.; Barker, D.J.; Bleker, O.P. Effects of prenatal exposure to the Dutch famine on adult disease in later life: An overview. Mol. Cell. Endocrinol. 2001, 185, 93-98. [CrossRef]

5. Barker, D.J. Fetal nutrition and cardiovascular disease in later life. Br. Med. Bull. 1997, 53, 96-108. [CrossRef] [PubMed]

6. Remacle, C.; Bieswal, F.; Bol, V.; Reusens, B. Developmental programming of adult obesity and cardiovascular disease in rodents by maternal nutrition imbalance. Am. J. Clin. Nutr. 2011, 94, 1846S-1852S. [CrossRef] [PubMed]

7. Van Straten, E.M.E.; Bloks, V.W.; van Dijk, T.H.; Baller, J.F.W.; Huijkman, N.C.A.; Kuipers, I.; Verkade, H.J.; Plösch, T. Sex-dependent programming of glucose and fatty acid metabolism in mouse offspring by maternal protein restriction. Gend. Med. 2012, 9, 166-179.e13. [CrossRef] [PubMed]

8. Zheng, J.; Xiao, X.; Zhang, Q.; Yu, M. DNA methylation: The pivotal interaction between early-life nutrition and glucose metabolism in later life. Br. J. Nutr. 2014, 112, 1850-1857. [CrossRef] [PubMed]

9. Burdge, G.C.; Hanson, M.A.; Slater-Jefferies, J.L.; Lillycrop, K.A. Epigenetic regulation of transcription: A mechanism for inducing variations in phenotype (fetal programming) by differences in nutrition during early life? Br. J. Nutr. 2007, 97, 1036-1046. [CrossRef] [PubMed]

10. Hanson, M.; Godfrey, K.M.; Lillycrop, K.A.; Burdge, G.C.; Gluckman, P.D. Developmental plasticity and developmental origins of non-communicable disease: Theoretical considerations and epigenetic mechanisms. Prog. Biophys. Mol. Biol. 2011, 106, 272-280. [CrossRef] [PubMed]

11. Ganu, R.S.; Harris, R.A.; Collins, K.; Aagaard, K.M. Early origins of adult disease: Approaches for investigating the programmable epigenome in humans, nonhuman primates, and rodents. ILAR J. 2012, 53, 306-321. [CrossRef] [PubMed]

12. Otani, L.; Shirasaka, N.; Yoshizumi, H.; Murakami, T. The effects of maternal mild protein restriction on stroke incidence and blood pressure in stroke-prone spontaneously hypertensive rats (SHRSP). Biosci. Biotechnol. Biochem. 2004, 68, 488-494. [CrossRef] [PubMed]

13. Otani, L.; Sugimoto, N.; Kaji, M.; Murai, M.; Chang, S.-J.; Kato, H.; Murakami, T. Role of the renin-angiotensin-aldosterone system in the enhancement of salt sensitivity caused by prenatal protein restriction in stroke-prone spontaneously hypertensive rats. J. Nutr. Biochem. 2012, 23, 892-899. [CrossRef] [PubMed]

14. Otani, L.; Yasumatsu, T.; Murakami, M.; Hayashi, A.; Kimoto, K.; Murakami, T. Reduction of salt sensitivity in stroke-prone spontaneously hypertensive rats administered an AT1 receptor antagonist during suckling. Am. J. Hypertens. 2006, 19, 979-984. [CrossRef] [PubMed]

15. Suganami, T.; Mori, K.; Tanaka, I.; Mukoyama, M.; Sugawara, A.; Makino, H.; Muro, S.; Yahata, K.; Ohuchida, S.; Maruyama, T.; et al. Role of prostaglandin E receptor EP1 subtype in the development of renal injury in genetically hypertensive rats. Hypertension 2003, 42, 1183-1190. [CrossRef] [PubMed]

16. Narumiya, S.; Sugimoto, Y.; Ushikubi, F. Prostanoid receptors: Structures, properties, and functions. Physiol. Rev. 1999, 79, 1193-1226. [CrossRef] [PubMed]

17. Breyer, M.D.; Jacobson, H.R.; Breyer, R.M. Functional and molecular aspects of renal prostaglandin receptors. J. Am. Soc. Nephrol. 1996, 7, 8-17. [PubMed]

18. Altmann, S.; Murani, E.; Schwerin, M.; Metges, C.C.; Wimmers, K.; Ponsuksili, S. Dietary protein restriction and excess of pregnant German Landrace sows induce changes in hepatic gene expression and promoter methylation of key metabolic genes in the offspring. J. Nutr. Biochem. 2013, 24, 484-495. [CrossRef] [PubMed]

19. Zeng, Y.; Gu, P.; Liu, K.; Huang, P. Maternal protein restriction in rats leads to reduced PGC-1 $\alpha$ expression via altered DNA methylation in skeletal muscle. Mol. Med. Rep. 2013, 7, 306-312. [CrossRef] [PubMed]

20. Suzuki, T.; Yamashita, S.; Ushijima, T.; Takumi, S.; Sano, T.; Michikawa, T.; Nohara, K. Genome-wide analysis of DNA methylation changes induced by gestational arsenic exposure in liver tumors. Cancer Sci. 2013, 104, 1575-1585. [CrossRef] [PubMed]

21. Yang, X.; Han, H.; De Carvalho, D.D.; Lay, F.D.; Jones, P.A.; Liang, G. Gene body methylation can alter gene expression and is a therapeutic target in cancer. Cancer Cell 2014, 26, 577-590. [CrossRef] [PubMed]

22. Guyton, A.C.; Coleman, T.G.; Cowley, A.V; Scheel, K.W.; Manning, R.D.; Norman, R.A. Arterial pressure regulation. Overriding dominance of the kidneys in long-term regulation and in hypertension. Am. J. Med. 1972, 52, 584-594. [CrossRef] 
23. Gu, X.; Gu, D.; He, J.; Rao, D.C.; Hixson, J.E.; Chen, J.; Li, J.; Huang, J.; Wu, X.; Rice, T.K.; et al. Resequencing Epithelial Sodium Channel Genes Identifies Rare Variants Associated with Blood Pressure Salt-Sensitivity: The GenSalt Study. Am. J. Hypertens. 2018, 31, 205-211. [CrossRef] [PubMed]

24. Canessa, C.M.; Schild, L.; Buell, G.; Thorens, B.; Gautschi, I.; Horisberger, J.D.; Rossier, B.C. Amiloride-sensitive epithelial $\mathrm{Na}^{+}$channel is made of three homologous subunits. Nature 1994, 367, 463-467. [CrossRef] [PubMed]

25. Pácha, J.; Frindt, G.; Antonian, L.; Silver, R.B.; Palmer, L.G. Regulation of Na channels of the rat cortical collecting tubule by aldosterone. J. Gen. Physiol. 1993, 102, 25-42. [CrossRef] [PubMed]

26. Zhang, W.; Xia, X.; Reisenauer, M.R.; Rieg, T.; Lang, F.; Kuhl, D.; Vallon, V.; Kone, B.C. Aldosterone-induced Sgk1 relieves Dot1a-Af9-mediated transcriptional repression of epithelial $\mathrm{Na}^{+}$channel alpha. J. Clin. Investig. 2007, 117, 773-783. [CrossRef] [PubMed]

27. González, A.A.; Céspedes, C.; Villanueva, S.; Michea, L.; Vio, C.P. E Prostanoid-1 receptor regulates renal medullary alphaENaC in rats infused with angiotensin II. Biochem. Biophys. Res. Commun. 2009, 389, 372-377. [CrossRef] [PubMed]

(C) 2018 by the authors. Licensee MDPI, Basel, Switzerland. This article is an open access article distributed under the terms and conditions of the Creative Commons Attribution (CC BY) license (http:/ / creativecommons.org/licenses/by/4.0/). 